\title{
ARTICLE Neural and behavioral effects of oxytocin administration during theory of mind in schizophrenia and controls: a randomized control trial
}

\author{
Lize De Coster ${ }^{1,2,3,4}$, Lisa Lin ${ }^{1,2,3}$, Daniel H. Mathalon ${ }^{1,2,3}$ and Joshua D. Woolley ${ }^{1,2,3}$
}

Social cognitive impairments, including theory of mind (ToM), in schizophrenia more strongly predict functional outcomes than psychotic symptoms or nonsocial cognitive deficits. Despite their clinical importance, current medications do not improve these deficits. The current study investigated the hypothesis that oxytocin, a neuropeptide implicated in social behavior, would normalize neural abnormalities in schizophrenia during ToM, and that this normalization would correlate improvement in ToM behavior. In this cross-over, double-blind, and placebo-controlled functional magnetic resonance imaging study, a single dose of $40 \mathrm{IU}$ of oxytocin was administered via nasal spray to male individuals with a schizophrenia spectrum disorder (schizophrenia and schizoaffective disorder, $n=23)$ and healthy controls $(n=25)$. Participants completed two ToM tasks in the scanner, the False Belief and Person Description tasks. During both tasks, on placebo day, schizophrenia was associated with reduced accuracy, hypo-activity in the right temporo-parietal junction (rTPJ; extended into the posterior superior temporal sulcus), and hypo-connectivity between the rTPJ and medial prefrontal cortex (mPFC) compared to healthy controls. Oxytocin, relative to placebo, significantly increased accuracy and rTPJ activation for ToM but not control stories in schizophrenia. Furthermore, a significant positive correlation was found between oxytocin induced increases in rTPJ activity and accuracy, indicating that oxytocin improved rTPJ activity in schizophrenia predicted behavioral improvement. Oxytocin also significantly improved connectivity between rTPJ and mPFC in schizophrenia. These findings suggest that rTPJ activity during ToM might be a potential neural target for the treatment of social cognitive deficits in schizophrenia.

Neuropsychopharmacology (2019) 44:1925-1931; https://doi.org/10.1038/s41386-019-0417-5

\section{INTRODUCTION}

Social cognitive impairments in schizophrenia more strongly predict functional outcomes than psychotic symptoms or nonsocial cognitive deficits [1]. Theory of mind (ToM), i.e., highlevel inferences about mental states [2], is a core social cognitive function deficient in schizophrenia that is associated with realworld social behavior and poor outcomes [1, 3-5]. Hypoactivity of a neural network, including the right temporo-parietal junction (rTPJ) and medial prefrontal cortex (mPFC), likely underlies these ToM deficits [6-11]. Despite their clinical importance, current antipsychotic medications do not improve ToM deficits or their underlying network pathophysiology [12].

Intranasally administered oxytocin can improve social cognitive, including ToM, deficits in schizophrenia [13-23]. However, results of both single-dose and chronic administration trials have been mixed $[22,23]$. The NIMH recommends that the first step in developing a new intervention is to demonstrate that it exerts a measurable effect on a hypothesized "target" mechanism of action, i.e., "target engagement", with subsequent trials to show that target engagement correlates with improvement in clinical outcomes $[22,24]$. However, to date, oxytocin administration studies in schizophrenia have focused on social cognitive outcomes without demonstration of target engagement, rendering the interpretation of negative results ambiguous.

We addressed this limitation by using functional magnetic resonance imaging (fMRI) to directly assess oxytocin's engagement of neurophysiological mechanisms subserving ToM, and its ability to remediate the neurophysiological abnormalities underlying ToM deficits in schizophrenia. fMRI studies examining oxytocin's neurophysiological effects in schizophrenia have focused on emotional cue perception, revealing that oxytocin attenuates activity in the amygdala [25], inferior temporal, fusiform, parahippocampal, middle frontal, and anterior cingulate gyri, and premotor cortex, and increases activity in the middle occipital, inferior occipital, and superior temporal gyri [26] during facial emotion recognition. The current study is the first to examine oxytocin's effects on neurophysiological activity during ToM in schizophrenia. In addition, we examined whether oxytocin-induced neurophysiological changes correlated with improvements in ToM performance.

We used two well-validated verbal ToM tasks [27-29] requiring prediction of a person's behavior based on his mental state [30]; the False Belief task (FBT) [31] and Person Description task (PDT) [32]. Both tasks have been reliably used to show that-in healthy controls-regions including the rTPJ, left temporo-parietal

\footnotetext{
${ }^{1}$ University of California, San Francisco, CA, USA; ${ }^{2}$ San Francisco Veterans Affairs Medical Center, San Francisco, CA, USA; ${ }^{3}$ UCSF Weill Institute for Neurosciences, San Francisco, CA, USA and ${ }^{4}$ Universidad Carlos III de Madrid, Madrid, Spain

Correspondence: Joshua D. Woolley (josh.woolley@ucsf.edu)
}

Received: 16 January 2019 Revised: 8 April 2019 Accepted: 7 May 2019

Published online: 18 May 2019 
junction (ITPJ), mPFC, and posterior cingulate $(\mathrm{pC})$ are activated during ToM [31, 32]. Furthermore, individuals with schizophrenia show impaired performance and hypoactivation in bilateral TPJ and mPFC compared to healthy controls $[10,11]$. Two different tasks were used to allow for internal replication. Furthermore, while the FBT has been used to measure cognitive ToM only, the PDT has been thought to reflect both cognitive and emotional ToM [10], allowing us to disentangle the effect of oxytocin on both types of ToM. In our study, we first compared activation for healthy controls and individuals with schizophrenia on placebo to identify and replicate brain activation abnormalities in schizophrenia. Subsequently, we examined the effect of oxytocin in schizophrenia in the regions showing aberrant activation compared to healthy controls. The current study investigated oxytocin's effect on pathophysiological mechanisms underlying ToM deficits in schizophrenia, and whether normalization of these mechanisms correlated with improvement in ToM.

\section{MATERIALS AND METHODS}

Participants

Participants were clinically stable male outpatients with a schizophrenia spectrum disorder (SZ; $n=23$ ) and healthy controls $(\mathrm{HC} ; n=25)$. For demographic data, see Table 1. Given the sexually dimorphic effects of oxytocin administration [33], only males were recruited to minimize intersubject variation. Participants provided written consent for the study after the experimenter made sure the participant understood all aspects of the study, and was able to read the informed consent form. The study was approved by the UCSF Institutional Review Board, and all experiments were performed in accordance with guidelines provided by the Committee on Human Research at UCSF. Participants completed all study interventions and tasks at the San Francisco Veterans Affairs Medical Center, with a physician on site to ensure participant's safety. See Supplementary Materials and Methods for further details.

\section{Procedure}

We used a randomized, double-blind, placebo-controlled, crossover design, with the two test days separated by at least 2 weeks. On each test day, oxytocin (40 IU, Wellspring Pharmacy, Berkeley) or placebo was administered by staff via nasal spray [34].

\begin{tabular}{|c|c|c|c|c|c|}
\hline & \multicolumn{2}{|c|}{$\begin{array}{l}\text { Healthy } \\
\text { Controls }\end{array}$} & \multicolumn{2}{|c|}{ Schizophrenia } & \multirow[t]{2}{*}{$p$-value } \\
\hline & Mean & SD & Mean & SD & \\
\hline$N$ & 25 & - & 23 & - & - \\
\hline Age & 27.96 & 6.98 & 35.30 & 10.48 & 0.01 \\
\hline Education & 15.38 & 2.26 & 15.30 & 1.99 & 0.91 \\
\hline WRAT & 61.84 & 4.86 & 61.17 & 4.30 & 0.62 \\
\hline AMNART & 117.74 & 9.87 & 117.57 & 8.13 & 0.95 \\
\hline ECR-RS avoidance & 2.65 & 1.17 & 2.60 & 0.97 & 0.86 \\
\hline ECR-RS anxiety & 1.52 & 0.62 & 2.30 & 0.99 & $<0.001$ \\
\hline PANSS positive & - & - & 11.56 & 3.98 & - \\
\hline PANSS negative & - & - & 12.13 & 4.73 & - \\
\hline PANSS general & - & - & 21.96 & 4.64 & - \\
\hline CPZ equivalent & - & - & 908.49 & 1964.96 & - \\
\hline
\end{tabular}

WRAT wide range achievement test, AMNART American version of the national adult reading test, $E C R-R S$ Experiences in close relationships scores-relationships structures, PANSS positive and negative syndrome scale, CPZ chlorpromazine
Experimental fMRI tasks (FBT followed by PDT) started 45 min after drug administration.

During the FBT $[11,31,35]$, participants read two kinds of short stories each presented for $17 \mathrm{~s}$. In Belief stories, a protagonist has a false belief about the current status of reality based on incomplete information about how reality has changed. In Photograph stories, a photograph contains an outdated depiction of reality that conflicts with current reality. In both cases, the participants were presented with a statement at the end of the story describing the protagonist's belief or the photograph's content, and indicated whether this was true or false by pressing one of two buttons on a response pad during the last $6 \mathrm{~s}$. Only Belief stories required ToM inferences. Between trials, a fixation cross appeared for $12 \mathrm{~s}$. Duration for FBT was 9.67 min (excluding breaks), consisting of 20 stories in two runs, with five Belief and five Photograph stories per run.

During the PDT $[10,32]$, three different types of short stories were presented for $15 \mathrm{~s}$ each, describing a protagonist's: (1) Thoughts (cognitive ToM), (2) Emotions (emotional ToM), or (3) Appearance. After each story, participants were presented with a statement about the protagonist's thoughts, emotions, or appearance and judged the accuracy of the statement by indicating yes/no on a response pad during the last $5 \mathrm{~s}$. Between trials, a fixation cross appeared for $12 \mathrm{~s}$. Duration for PDT was 13.5 min, consisting of 30 stories divided over five runs, with two Thought, two Emotion, and two Appearance stories per run. See Supplementary Materials and Methods for example stories for both tasks. Materials and information can be obtained from the researchers upon request.

\section{Data analysis}

The primary outcome was oxytocin-induced neural changes in SZ in regions showing abnormalities on Placebo day compared to $\mathrm{HC}$. Secondary outcomes included behavioral and connectivity changes, and correlations between oxytocin-induced neural and behavioral changes.

Behavior. Task performance accuracy (\% correct) was analyzed using a repeated measures ANOVA with Condition (FBT: Belief versus Photograph; PDT: Thought versus Emotion versus Appearance) and Drug (Oxytocin versus Placebo) as within-subjects factors in both groups. Significant interactions were parsed by testing lower-order simple effects. See Supplementary Materials and Methods for reaction time analyses.

Image acquisition and preprocessing. MRI images were acquired with a 3T Siemens Skyra scanner with a 32-channel radiofrequency head coil. Scanning started with a prepared rapid gradient-echo T1-weighted high-resolution MPRAGE sequence (TR $=2300 \mathrm{~ms}$, $\mathrm{TE}=2.98 \mathrm{~ms}$, slice thickness $=1.20 \mathrm{~mm}$, field of view $=256 \mathrm{~mm}$, voxel size $=1.0 \times 1.0 \times 1.2 \mathrm{~mm}$, flip angle $\left.=9^{\circ}\right)$. Next, whole-brain functional images were conducted using a $\mathrm{T} 2^{*}$-weighted echo planar imaging (EPI) sequence, sensitive to blood-oxygen-level dependent (BOLD) contrast $(\mathrm{TR}=2000 \mathrm{~ms}$, $\mathrm{TE}=30 \mathrm{~ms}$, voxel size $=3.4 \times 3.4 \times 4.0 \mathrm{~mm}$, flip angle $=77^{\circ}, 30$ slices collected in ascending order). The amount of EPI images for the FBT was 278 and for the PDT was 375. Data were high pass filtered at $128 \mathrm{~s}$ during analysis.

Functional analysis. Single subject fMRI scan time-series data, comprising concatenated runs and test days, were slice-time corrected, realigned, and normalized to the Montreal Neurological Institute EPI template, and smoothed $(8 \mathrm{~mm})$ using SPM8 (Wellcome Department of Imaging Neuroscience, UCL, London, UK). SPM's general linear model was applied to single voxel BOLD signal time-series data. For each task separately, the entire trial was modeled (story and judgment) using generalized least squares with a global approximate $\mathrm{AR}(1)$ autocorrelation model. 

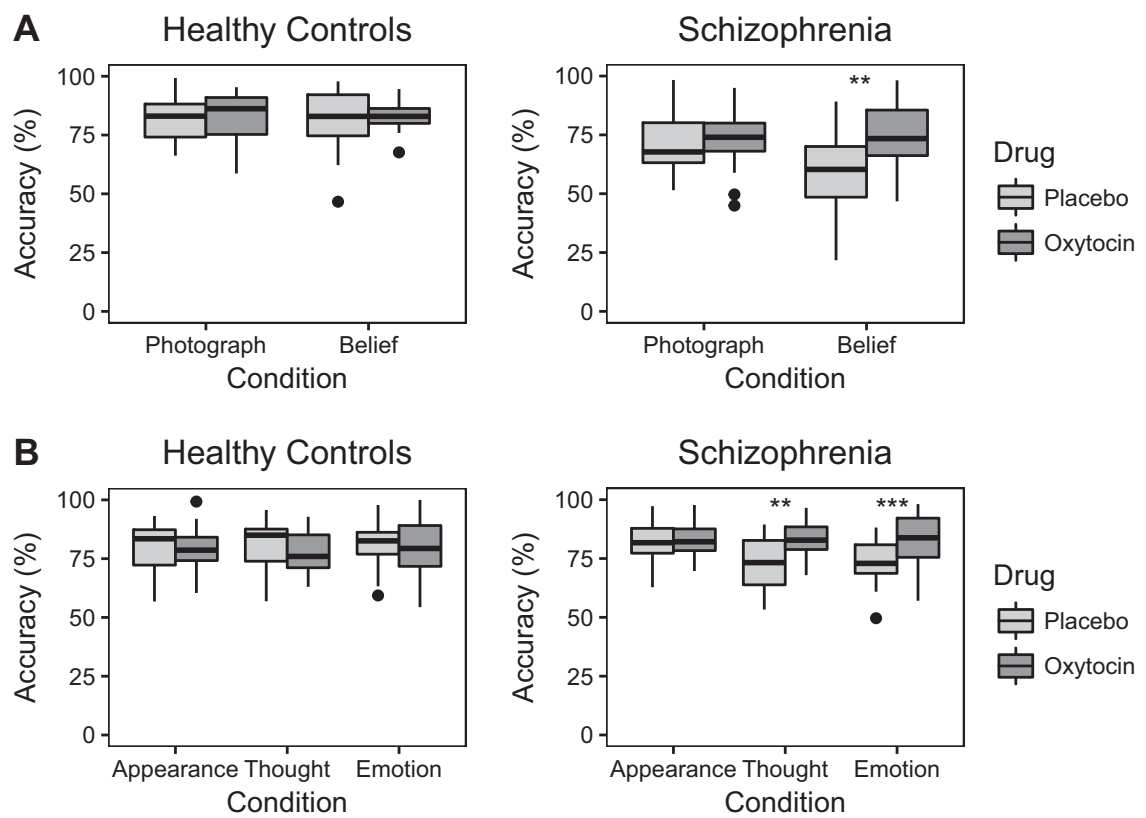

Fig. 1 \% Accuracy in a the False Belief Task and $\mathbf{b}$ the Person Description Task for healthy controls $(n=25)$ and individuals with schizophrenia $(n=23)$. Thick black line within the boxplot represents the median, the box indicates the interquartile range, and the whiskers represent minimum and maximum. Single dots indicate outliers. ${ }^{* *} p<0.01,{ }^{* * *} p<0.001$

The model included a separate regressor for each task condition and drug test day (FBT: Photograph-Placebo, Belief-Placebo, Photograph-Oxytocin, Belief-Oxytocin; PDT: Thought-Placebo, Emotion-Placebo, Thought-Oxytocin, Emotion-Oxytocin) convolved with SPM's canonical hemodynamic response function. In addition, six motion parameter nuisance regressors were included to control for head movements. Behavioral responses were not modeled (see also [11, 32]). Parameter estimates for each task and drug condition were subtracted to generate first-level contrast images for comparisons of interest. Both test days were modeled in a single first-level model to reduce warping errors.

To identify brain activation abnormalities in SZ relative to $\mathrm{HC}$, whole-brain voxel-wise independent samples $t$-tests were performed to compare the groups on Placebo day contrasts (FBT: Belief-Photograph, PDT: Thought-Appearance, Emotion-Appearance). Regions showing significant SZ abnormalities were subsequently interrogated in region of interest (ROI) analyses based on the activated clusters in the contrasts of interest on Placebo day to assess the effects of oxytocin in SZ. Mean beta weights were extracted from each ROI for each task condition and drug test day in SZ and were subsequently entered into a two-way repeated measures ANOVA with Condition (FBT: Belief versus Photograph; PDT: Thought versus Emotion versus Appearance) and drug (Oxytocin versus Placebo) as within-subjects factors. See Supplementary Materials and Methods for additional exploratory wholebrain analyses.

Correlations. Oxytocin-induced changes in task performance were correlated with the oxytocin-induced changes in mean ROI task contrast values within SZ. Correlations were corrected for multiple comparisons $(n=3)$ with false discovery rate correction using the p.adjust function in R [36]. See Supplementary Materials and Methods for additional correlations.

Connectivity. To examine task- and drug-related changes in functional connectivity within the ToM network, psychophysiological interaction (PPI) analyses were conducted using the generalized psychophysiological interaction (gPPI) toolbox [37] in four critical nodes of the ToM network previously identified using a ToM localizer task [32]: rTPJ, ITPJ, mPFC, and pC. gPPI models were run for each of the six unique node pairs, separately for each participant, test day, and task. PPI values for task condition contrasts were compared in (1) SZ and HC on the Placebo day, and (2) Oxytocin and Placebo days within SZ. See Supplemental Material for further details.

Statistical thresholds. For behavioral and ROI analyses, alpha was set to $p<0.05$, two-tailed. ANOVA and lower-order $t$-tests of behavioral, ROI, and PPI data were not corrected for multiple comparisons given our a priori hypotheses. For all whole-brain voxel-wise analyses, statistical significance was based on a voxelwise height threshold of $p<0.001$ followed by a family-wise error-corrected cluster-level significance threshold of $p<0.05$, two-tailed (minimum cluster size $k>86$ ).

\section{RESULTS}

Behavior

FBT. In SZ, a Condition $\times$ Drug interaction $(F(1,22)=5.90$, $p=0.02, \eta_{p}^{2}=0.21$ ) indicated that Oxytocin improved accuracy for Belief $(M=75.54 \%, S D=14.29 \%)$ but not Photograph stories $(M=72.99 \%, S D=11.69 \%)$, compared to Placebo (Belief: $M=59.29 \%, S D=16.99 \%, t(22)=3.38, p=0.003$, and $d=1.06$; Photograph: $M=70.39 \%, S D=11.89 \%, t(22)=0.75$, and $p=0.46)$. The Condition $\times$ Drug interaction was not significant in $\mathrm{HC}(F(1,24)$ $=0.06, p=0.81$ ). See Fig. 1 a.

PDT. In SZ, a Condition $\times$ Drug interaction $(F(2,21)=6.31, p=$ $\left.0.007, \eta_{p}^{2}=0.38\right)$ indicated that Oxytocin improved accuracy for Thought $(M=82.95 \%, S D=7.98 \%)$ and Emotion $(M=83.22 \%$, $S D=10.36 \%)$ but not for Appearance stories $(M=83.33 \%, S D=$ $8.34 \%$ ), relative to Placebo (Thought: $M=73.37 \%, S D=10.94 \%$, $t(22)=3.61, p=0.002$, and $d=0.72$; Emotion: $M=71.50 \%, S D=$ $10.31 \%, t(22)=3.80, p<0.001$, and $d=0.76$; Appearance: $M=$ $82.22 \%, S D=8.95 \%, t(22)=0.50, p=0.62)$. The Condition $\times$ Drug interaction was not significant in $\mathrm{HC}(F(2,23)=0.15, p=0.86)$. See Fig. $1 b$. 
A
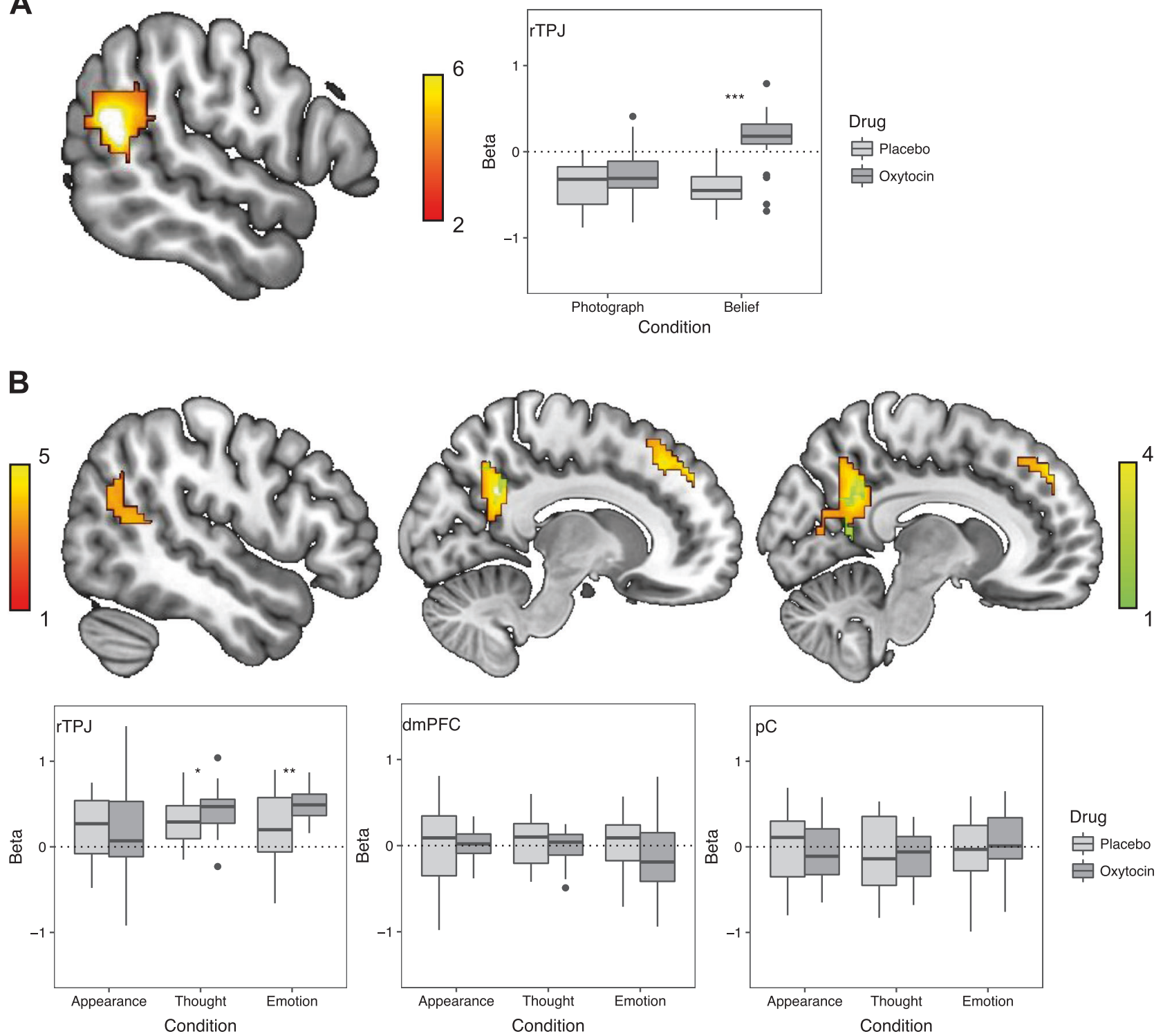

Drug

官 Placebo

官 Oxytocin

Fig. 2 a Whole-brain contrasts comparing healthy controls $(n=25)$ and individuals with schizophrenia $(n=23)$ on placebo day for the False Belief Task (FBT; Belief-Photograph). Region of interest (ROI) analyses in individuals with schizophrenia ( $n=23)$ in the FBT using the ROI (right temporo-parietal junction; rTPJ; $57-5421$ ) defined in the whole-brain contrast. b Whole-brain contrasts comparing healthy controls ( $n=25$ ) and individuals with schizophrenia $(n=23)$ on placebo day for the Person Description Task (PDT; orange: Thought-Appearance, green: Emotion-Appearance). ROI analyses in individuals with schizophrenia $(n=23)$ in the PDT using the ROls (rTPJ; $63-48$ 12, right dorsal medial prefrontal cortex; rdmPFC; 1251 42, posterior cingulate; pC; 12 -51 33) defined in the whole-brain contrast. Thick black line within the boxplot represents the median, the box indicates the interquartile range, and the whiskers represent minimum and maximum. Single dots indicate outliers. ${ }^{*} p<0.05,{ }^{* *} p<0.01,{ }^{* * *} p<0.001$

\section{Imaging}

FBT. The Belief-Photograph whole-brain contrast on the Placebo day revealed hypoactivation in SZ relative to HC in the rTPJ (57 $-5421, z=6.00, n=383, p<0.001$; see Fig. 2a). Activation within this cluster was extracted as an ROI for additional analyses in SZ.

Within this rTPJ ROI, a Condition $\times$ Drug interaction $(F(1,22)=$ $35.49, p<0.001, \eta_{p}{ }^{2}=0.62$ ) emerged (see Fig. 2a), with follow-up $t$-tests indicating that Oxytocin $(M=0.14, S D=0.34)$, relative to Placebo $(M=-0.41, S D=0.22)$, increased rTPJ activation for Belief $(t(22)=8.54, p<0.001$, and $d=0.96)$ but not Photograph stories (Oxytocin: $M=-0.24, S D=0.31$; Placebo: $M=-0.37, S D=0.27$; $t(22)=1.87, p=0.08)$.

$P D T$. The Thought-Appearance whole-brain contrast on the Placebo day revealed hypoactivation in SZ, relative to $\mathrm{HC}$, in rTPJ
(63 $-4812, z=4.21, n=165$, and $p=0.004)$, right dorsal mPFC (12 $5142, z=4.39, n=181$, and $p=0.002)$, and $\mathrm{pC}(12-5133$, $z=4.55, n=519$, and $p<0.001)$. The whole-brain contrast (Emotion-Appearance) on the Placebo day revealed hypoactivation for SZ, relative to HC, in $\mathrm{pC}(6-4836, z=3.58, n=136$, and $p=0.02$; see Fig. $2 b$ ). Activation within these clusters were extracted as ROIs for additional analyses in SZ.

In the rTPJ ROI only, a Condition $\times$ Drug interaction was observed $\left(F(2,21)=4.42, p=0.03\right.$, and $\eta_{p}^{2}=0.30$; see Fig. $\left.2 b\right)$. Thought and Emotion stories elicited greater activation on the Oxytocin (Thought: $M=0.42, S D=0.26$; Emotion: $M=0.50$, $S D=0.17$ ) compared to Placebo (Thought: $M=0.29, S D=0.27$, $t(22)=2.30, p=0.03$, and $d=0.50$; Emotion: $M=0.21, S D=0.42$, $t(22)=3.11, p=0.005$, and $d=0.93)$ days, but Appearance 


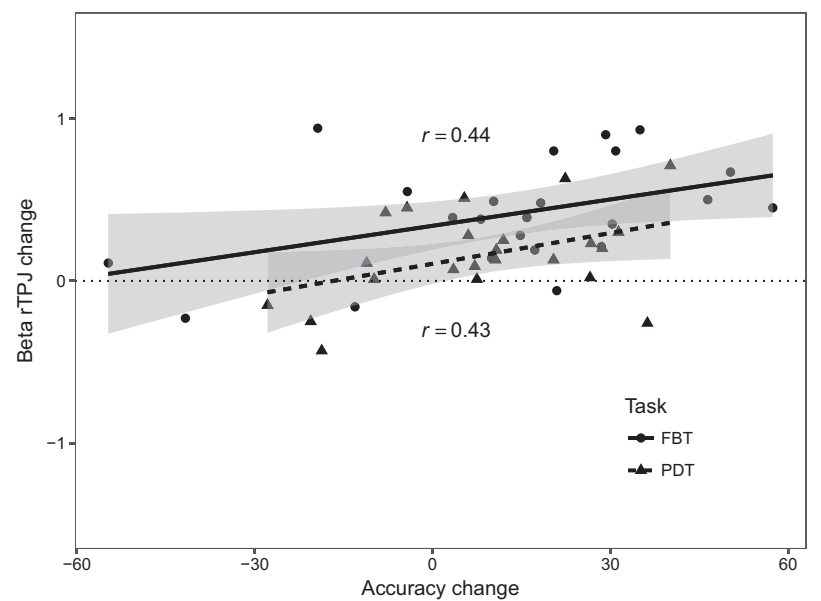

Fig. 3 Correlations between accuracy change scores in the False Belief Task (FBT) and Person Description Task (PDT) and right temporoparietal junction (rTPJ) beta weights comparing Oxytocin and Placebo days using the contrasts [(Belief Oxytocin-Photograph Oxytocin) (Belief Placebo-Photograph Placebo)] for FBT and [(Thought Oxytocin-Appearance Oxytocin)-(Thought Placebo-Appearance Placebo)] for PDT in individuals with schizophrenia $(n=23)$

stories did not (Oxytocin: $M=0.19, S D=0.62$; Placebo: $M=0.20$, $S D=0.37 ; t(22)=0.18$, and $p=0.86)$.

\section{Correlations}

Given that oxytocin only changed behavior in SZ, correlations concerning these changes were only computed within this group. In the FBT, oxytocin-induced activity increase in the rTPJ ROI for the [(Belief Oxytocin-Photograph Oxytocin)-(Belief Placebo-Photograph Placebo)] contrast and oxytocin-induced increase in accuracy for the same contrast were positively correlated $(r=0.44, p=0.04)$. In the PDT, a similar positive correlation between oxytocin-induced activity increases in the rTPJ ROI for the [(Thought Oxytocin-Appearance Oxytocin) (Thought Placebo-Appearance Placebo)] contrast and the oxytocin-induced accuracy increases was observed $(r=0.43$, $p=0.04$; see Fig. 3 ).

\section{Connectivity}

FBT. The PPI analysis on Placebo day using the rTPJ-mPFC connectivity pair revealed a Condition $\times$ Group interaction $\left(F(1,46)=41.24, p<0.001\right.$, and $\eta_{p}^{2}=0.47$; see Fig. 4a). In $\mathrm{HC}$, greater connectivity was observed during Belief $(M=0.67, S D=$ $0.17)$ than during Photograph $(M=0.17, S D=0.15 ; t(24)=10.46$, $p<0.001$, and $d=0.82$ ) stories, while this difference was not significant in SZ $(t(22)=0.48, p=0.64)$.

When comparing Oxytocin and Placebo days, a Condition $x$ Drug interaction for the rTPJ-mPFC connectivity pair was observed in SZ $\left(F(1,22)=7.03, p=0.02\right.$, and $\eta_{p}{ }^{2}=0.24$; see Fig. 4b). During the Oxytocin day only, greater connectivity was observed for Belief $(M=0.63, S D=0.24)$ than for Photograph stories $(M=0.30, S D=0.30 ; t(22)=3.61, p=0.002$, and $d=0.92$ ).

PDT. The PPI analysis on Placebo day using the rTPJ-mPFC connectivity pair revealed a Condition $\times$ Group interaction $(F$ $(2,45)=4.48, p=0.02$, and $\eta_{p}{ }^{2}=0.17$; see Fig. $\left.4 \mathrm{C}\right)$. In HC, greater functional connectivity was observed during Thought stories $(M=0.55, S D=0.25)$ compared to Appearance $(M=0.18, S D=$ $0.28 ; t(24)=4.28, p<0.001$, and $d=0.84)$ and Emotion stories $(M=0.18, S D=0.39 ; t(24)=3.66, p=0.001$, and $d=0.75)$, while these differences were not significant in SZ $(t(22)=0.13, p=$ 0.90 and $t(22)=0.39$, and $p=70)$.
Comparing Oxytocin and Placebo days in SZ, a Condition $\times$ Drug interaction for the rTPJ-mPFC connectivity pair approached significance $\left(F(2,21)=3.19, p=0.06\right.$, and $\eta_{p}{ }^{2}=$ 0.23; see Fig. 4d). Greater connectivity was observed for Thought $(M=0.40, S D=0.33)$ than for Appearance $(M=0.08$, $S D=0.40 ; t(22)=3.09, p=0.005$, and $d=0.89)$ and Emotion stories $(M=-0.01, S D=0.49 ; t(22)=3.62, p=0.002$, and $d=$ $0.90)$ on the Oxytocin day only.

No other PPI effects were significant.

\section{DISCUSSION}

We found that during ToM [10, 11], schizophrenia is associated with hypoactivity in a neural network including the rTPJ and decreased functional connectivity between the rTPJ and mPFC. In addition, we found that a single dose of oxytocin increases rTPJ activity, improves ToM accuracy, and strengthens connectivity between the rTPJ and the MPFC during ToM in schizophrenia. We also observed heterogeneity in responses to oxytocin [22]. Specifically, the magnitude of oxytocin-induced increase in rTPJ activity correlated with the magnitude of oxytocin-induced improvements in ToM behavioral performance in schizophrenia. Furthermore, these results were consistent across two wellvalidated ToM tasks [31, 32]. Together, these data suggest that oxytocin-induced increases in rTPJ activity may underlie oxytocininduced ToM improvement in schizophrenia. Thus, ToM-related rTPJ activity might be a useful measure of target engagement for future studies of oxytocin to better target the underlying mechanisms of social deficits in schizophrenia.

Our findings suggest that oxytocin may influence a distributed ToM-related network that is responsible for the attribution of thoughts to another person (rTPJ) and representing emotionally relevant information about another person (mPFC) $[32,38,39]$. Interestingly, results of the whole-brain comparison between SZ and $\mathrm{HC}$ on Placebo day for FBT partially extended into the posterior superior temporal sulcus (pSTS). The pSTS has been related to social cognition [40], including ToM in schizophrenia [41]. Given the link between ToM and social functioning in schizophrenia [1, 3-5], our finding that oxytocin improves the neural activity underlying ToM behavior in schizophrenia is potentially clinically meaningful. The degree to which oxytocin normalizes the neural substrates underlying ToM dysfunction (particularly the rTPJ) may predict the likelihood that an individual's social functioning will benefit from longer-term oxytocin treatment. This would allow for more consistent clinical trials and personalized treatments, which is important given current difficulties in translating basic research to clinical practice [22-24]. Indeed, unaccounted for heterogeneity in target engagement by oxytocin administration may also help explain the mixed results in previous repeated administration trials in schizophrenia. Interestingly, a recent meta-analysis [42] indicated that individuals with schizophrenia show a simultaneous hypoactivation of the posterior ITPJ and hyperactivation of the bilateral dorsal temporal junction, and increased activation in attention-related networks that most likely reflect compensatory mechanisms. Given that oxytocin has recently been shown to modulate the intrinsic dynamics of attention-related networks [43], these findings suggest that future studies should look into the effects of oxytocin on attention networks in schizophrenia.

The current study has several limitations. First, given our relatively small all-male sample, our findings warrant replication, as well as verification of the test-retest reliability of these effects. This is warranted given the inconsistent findings from fMRI studies on oxytocin and social cognition. However, our withinsubject design and the consistency of results in two related but distinct tasks are strong advantages of the current study. Second, not all effects were corrected for multiple comparisons (e.g., lower-order $t$-tests). While not all effects met multiple correction 


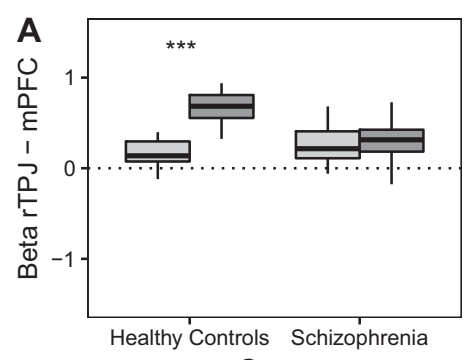

Group

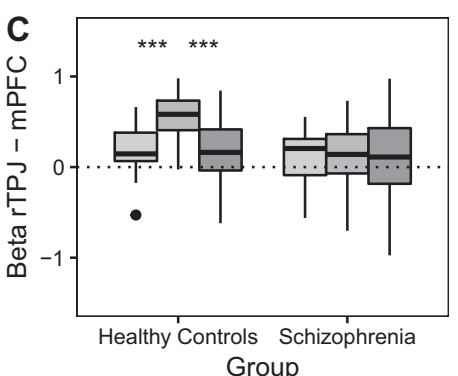

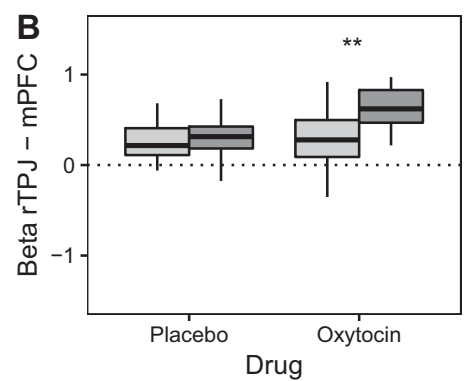

Condition

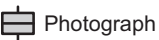

Belief

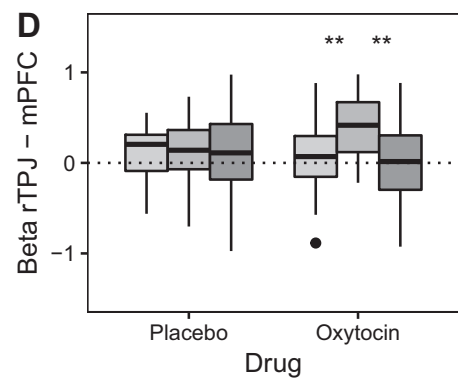

Condition

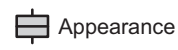

官 Thought

它 Emotion

Fig. 4 Results of the psychophysiological interaction analyses using the right temporo-parietal junction (rTPJ; 53 -54 17), left temporoparietal junction $(-48-5725)$, medial prefrontal cortex (mPFC; 1632$)$, and posterior cingulate $(2-6027)$ as seeds. a Functional connectivity changes between the rTPJ and the mPFC on placebo day comparing healthy controls $(n=25)$ and individuals with schizophrenia $(n=23)$ in the False Belief task (FBT). b Comparing connectivity on Oxytocin and Placebo days between the rTPJ and mPFC in schizophrenia ( $n=23$ ) in the FBT. c Functional connectivity changes between the rTPJ and the mPFC on Placebo day comparing healthy controls $(n=25)$ and individuals with schizophrenia $(n=23)$ in the Person Description Task (PDT). d Comparing connectivity on Oxytocin and Placebo days between the rTPJ and mPFC in schizophrenia $(n=23)$ in the PDT. Thick black lines within the boxplot represents the median, the box indicates the interquartile range, and the whiskers represent minimum and maximum. Single dots indicate outliers. ${ }^{* *} p<0.01,{ }^{* * *} p \leq 0.001$

levels of significance, we believed that these trends were worth investigating given our hypotheses (i.e., that oxytocin would increase activation in areas that show abnormalities on placebo day in schizophrenia compared to healthy controls). Third, linking ToM-induced neural and behavioral changes to other measures of social functioning is needed. Repeated administration trials of oxytocin in schizophrenia have been mixed and there are many reasons why acute effects may be found under ideal laboratory conditions but more chronic effects with individuals self-administering the drug at home could be different (e.g., temporal dynamics, adherence monitoring, etc. [44]). Future repeated administration clinical trials of oxytocin should include the rTPJ measure of target engagement to help elucidate these issues. Fourth, no blood oxytocin levels, as an index of plasma level of oxytocin after intranasal administration, were measured. However, plasma levels of oxytocin are not necessarily correlated with levels of oxytocin in cerebrospinal fluid, with the latter in all likelihood being the source of effects on cognition and behavior [45]. While it is questioned whether intranasal oxytocin administration can deliver enough oxytocin to the brain with only modest increases in cerebrospinal fluid oxytocin levels [46], these increases might still be functionally relevant [47]. Fifth, oxytocin-induced changes in the PDT were more consistent for Thought than for Emotion stories. Belief and Thought stories are both considered to reflect cognitive ToM, while Emotion stories reflect affective ToM [10], and the rTPJ is only activated during cognitive ToM [32]. Because our results suggest that oxytocin might have a strong influence on rTPJ activation, this could indicate that oxytocin selectively influences cognitive ToM. Sixth, the individuals with schizophrenia in the current study are relatively well-educated with high premorbid cognitive functioning and low current symptom severity. While this may decrease the generalizability of our results, it also means that our results are less likely to be confounded by differences in crystalized intelligence, educational attainment, low socioeconomic status, or long-stretches of social isolation or poor functioning that can be common in schizophrenia. Seventh, future research is needed to explore the influence of individual and contextual factors [22] that have been shown to influence oxytocin's effects on behavioral [48] and neural responses [49]. Finally, in this and our previous studies, oxytocin effects were only present in the schizophrenia group [21]. Given that healthy controls are typically not impaired in ToM, however, this was not unexpected [48].

This pharmaco-fMRI study demonstrates that oxytocin administration increases activity in ToM-related brain areas, particularly the rTPJ, in schizophrenia. Furthermore, oxytocin-induced activity increases correlate with oxytocin-induced behavioral improvement. These results suggest that rTPJ activity during ToM is a potential neural target for the treatment of social cognitive deficits in schizophrenia; an essential first step in determining a mechanism of target engagement for oxytocin treatment in schizophrenia. Further research is needed to translate these findings to real-world social behavior and clinical outcomes in schizophrenia, and generalize these findings to other patient populations that show ToM abnormalities such as autism spectrum disorders [50].

\section{FUNDING AND DISCLOSURE}

This study was supported by the Veterans Health Administration Office of Research and Development Career Development Award 1IK2CX000758-01A1 awarded to JDW. The authors declare no competing interests.

\section{ACKNOWLEDGEMENTS}

The authors would like to thank Christine Hooker for providing the stimulus materials. 


\section{ADDITIONAL INFORMATION}

Supplementary Information accompanies this paper at (https://doi.org/10.1038/ s41386-019-0417-5)

Publisher's note: Springer Nature remains neutral with regard to jurisdictional claims in published maps and institutional affiliations.

\section{REFERENCES}

1. Fett AK, Viechtbauer W, Dominguez MD, Penn DL, van Os J, Krabbendam L. The relationship between neurocognition and social cognition with functional outcomes in schizophrenia: a meta-analysis. Neurosci Biobehav Rev. 2011;35:573-88.

2. Premack DG, Woodruff G. Does the chimpanzee have a theory of mind? Behav Brain Sci. 1978;1:515-26.

3. Couture SM, Granholm EL, Fish SC. A path model investigation of neurocognition, theory of mind, social competence, negative symptoms, and real-world functioning in schizophrenia. Schizophr Res. 2011;125:152-60.

4. Mancuso F, Horan WP, Kern RS, Green MF. Social cognition in psychosis: multidimensional structure, clinical correlates, and relationship with functional outcome. Schizophr Res. 2011;125:143-51.

5. Kurtz MM, Richardson CL. Social cognitive training for schizophrenia: a metaanalytic investigation of controlled research. Schizophr Bull. 2012;38:1092-104.

6. Brunet E, Sarfati Y, Hardy-Baylé MC. Reasoning about physical causality and other's intentions in schizophrenia. Cogn Neuropsychiatry. 2003;8:129-39.

7. Brüne MD. "Theory of Mind" in schizophrenia: a review of the literature. Schizophr Bull. 2005;31:21-42.

8. Lee $Y$, Quintana J, Nori $P$, Green MF. Theory of mind in schizophrenia: exploring neural mechanisms of belief attribution. Soc Neurosci. 2011;6:569-81.

9. Das P, Lagopoulos J, Coulston CM, Henderson AF, Malhi GS. Mentalizing impairment in schizophrenia: a functional MRI study. Schizophr Res. 2012;134:158-64.

10. Dodell-Feder D, DeLisi LE, Hooker Cl. Neural disruption to theory of mind predicts daily social functioning in individuals at familial high-risk for schizophrenia. Soc Cogn Affect Neurosci. 2014a;9:1914-25.

11. Dodell-Feder D, Tully LM, Lincoln SH, Hooker $\mathrm{Cl}$. The neural basis of theory of mind and its relationship to social functioning and social anhedonia in individuals with schizophrenia. Neuroimage Clin. 2014b;4:154-63.

12. Kucharska-Pietura K, Mortimer A. Can antipsychotics improve social cognition in patients with schizophrenia? CNS Drugs. 2013;27:335-43.

13. Kosfeld M, Heinrichs M, Zak PJ, Fischbacher U, Fehr E. Oxytocin increases trust in humans. Nature. 2005;435:673-6.

14. Baumgartner $T$, Heinrichs $M$, Volanthen A, Fischbacher U, Fehr E. Oxytocin shapes the neural circuitry of trust and trust adaptation in humans. Neuron. 2008;58:639-50.

15. Kéri S, Benedek G. Oxytocin enhances the perception of biological motion in humans. Cogn Affect Behav Neurosci. 2009;9:237-41.

16. Hurlemann R, Patin A, Onur OA, Cohen MX, Baumgartner $T$, Metzler $S$, et al. Oxytocin enhances amygdala dependent, socially reinforced learning and emotional empathy in humans. J Neurosci. 2010;30:4999-5007.

17. Marsh AA, Yu HH, Pine DS, Blair RJR. Oxytocin improves specific recognition of positive facial expressions. Psychopharmacology. 2010;209:225-32.

18. Perry A, Bentin S, Shalev I, Israel S, Uzefovsky F, Bar-On D, et al. Intranasal oxytocin modulates EEG mu/alpha and beta rhythms during perception of biological motion. Psychoneuroendocrinology. 2010;35:1446-53.

19. Pederson CA, Gibson CM, Rau SW, Salimi K, Smedley KL, Casey RL, et al. Intranasa oxytocin reduces psychotic symptoms and improves theory of mind and social perception in schizophrenia. Schizophr Res. 2011;132:50-3.

20. Davis MC, Lee J, Horan WP, Clarke AD, McGee MR, Green MF, et al. Effects of single dose intranasal oxytocin on social cognition in schizophrenia. Schizophr Res. 2013;147:393-7.

21. Woolley JD, Chuang B, Lam O, Lai W, O'Donovan A, Rankin KP, et al. Oxytocin administration enhances controlled social cognition in patients with schizophrenia. Psychoneuroendocrinology. 2014;47:116-25.

22. Bradley ER, Woolley JD. oxytocin effects in schizophrenia: reconciling mixed findings and moving forward. Neurosci Biobehav Rev. 2017;80:36-56.

23. Williams D, Bürkner P. Effects of intranasal oxytocin on symptoms of schizophrenia: a multivariate Bayesian meta-analysis. Psychoneuroendocrinology. 2017:75:141-51.
24. Insel TR. Translating oxytocin neuroscience to the clinic: a National Institute of Mental Health perspective. Biol Psychiatry. 2016;79:153-4.

25. Shin NY, Park HY, Jung WH, Park JW, Yun JY, Jang JH, et al. Effects of oxytocin on neural response to facial expressions in patients with schizophrenia. Neuropsychopharmacology. 2015;40:1919-27.

26. Dey A, Rao N. Effects of oxytocin on neural response to facial emotion recognition in schizophrenia. Schizophr Bull. 2017;43:S75-S76.

27. Bora $E$, Yucel $M$, Pantelis $C$. Theory of mind impairment in schizophrenia: metaanalysis. Schizophr Res. 2009;109:1-9.

28. Chung YS, Barch D, Strube M. A meta-analysis of mentalizing impairments in adults with schizophrenia and autism spectrum disorder. Schizophr Bull. 2013;40:602-16.

29. Roux P, Smith P, Passerieux C, Ramus F. Preserved implicit mentalizing in schizophrenia despite poor explicit performance: evidence from eye tracking. Sci Rep. 2016;6:1-9.

30. Wimmer $\mathrm{H}$, Perner J. Beliefs about beliefs: representation and constraining function of wrong beliefs in young children's understanding of deception. Cognition. 1983;13:103-28.

31. Saxe R, Kanwisher N. People thinking about thinking people: the role tempoparietal junction "theory mind". Neuroimage. 2003;19:1835-42.

32. Saxe R, Powell LJ. It's the thought that counts: specific brain regions for one component of theory of mind. Psychol Sci. 2006;17:692-9.

33. Meyer-Lindenberg A, Domes G, Kirsch P, Heinrichs M. Oxytocin and vasopressin in the human brain: social neuropeptides for translational medicine. Nat Rev Neurosci. 2011;12:524-38.

34. Feifel D, Macdonald K, Nguyen A, Cobb P, Warlan H, Galangue B, et al. Adjunctive intranasal oxytocin reduces symptoms in schizophrenia patients. Biol Psychiatry. 2010;68:678-80.

35. Dodell-Feder D, Koster-Hale J, Bedny M, Saxe R. fMRI item analysis in a theory of mind task. Neuroimage. 2011;55:705-12.

36. $R$ core team (2018). R: A language and environment for statistical computing. $R$ foundation for Statistical Computing, Vienna, Austria. https://www.R-project.org/.

37. McLaren DG, Ries ML, Xu G, Johnson SC. A generalized form of contextdependent psychophysiological interactions (gPPI): a comparison to standard approaches. Neuroimage. 2012;61:1277-86.

38. Saxe R, Wexler A. Making sense of another mind: the role of the right temporoparietal junction. Neuropsychologia. 2005;43:1391-9.

39. Moessnang C, Otto K, Bilek E, Schäfer A, Baumeister S, Hohmann S, et al. Differential responses to the dorsomedial prefrontal cortex and right posterior superior temporal sulcus to spontaneous mentalizing. Hum Brain Mapp. 2017;38:3791-803.

40. Hein G, Knight RT. Superior temporal sulcus-it's my area: or is it? J Cogn Neurosci. 2008;20:2125-36.

41. Mier D, Eisenacher S, Rausch F, Englisch S, Gerchen MF, Zamoscik V, et al. Aberrant activity and connectivity of the posterior superior sulcus during social cognition in schizophrenia. Eur Arch Psychiatry Clin Neurosci. 2017;267:597-610.

42. Kronbichler L, Tschernegg M, Martin Al, Schurz M, Kronbichler M. Abnormal brain activation during theory of mind tasks in schizophrenia: a meta-analysis. Schizophr Bull. 2017;43:1240-50.

43. Xin F, Zhou F, Zhou X, Ma X, Geng Y, Zhao W, et al. Oxytocin modulates the intrinsic dynamics between attention-related large-scale networks. Cerebral Cortex. 2018;bhy295. https://doi.org/10.1093/cercor/bhy295.

44. Djupesland PG. Nasal drug delivery devices: characteristics and performance in a clinical perspective-a review. Drug Deliv Transl Res. 2013;3:42-62.

45. Leng G, Ludwig M. Intranasal oxytocin: myths and delusions. Biol Psychiatry. 2016;79:243-50.

46. Quintana DS, Woolley JD. Intranasal oxytocin mechanisms can be better understood but its effects on social cognition and behavior are not to be sniffed at. Biol Psychiatry. 2016;79:e49-e50.

47. Neumann ID, Landgraf R. Balance of brain oxytocin and vasopressin: implications for anxiety, depression, and social behaviors. Trends Neurosci. 2012;35:649-59.

48. Bartz JA, Zaki J, Bolger N, Oschner KN. Social effects of oxytocin in humans: context and person matter. Trends Cogn Sci. 2011;15:301-9.

49. Shen H. Neuroscience: the hard science of oxytocin. Nature. 2015;522:410-2.

50. Aoki Y, Yahata N, Watanabe T, Takano Y, Kawakubo Y, Kuwabara H, et al. Oxytocin improves behavioral and neural deficits in inferring others' social emotions in autism. Brain. 2014;137:3073-86. 